\title{
Time Domain Equalization for OFDM Systems
}

\author{
Shaodan Ma, Ngai Wong, and Tung Sang Ng, Fellow, IEEE \\ Dept. of Electrical and Electronic Engineering \\ The University of Hong Kong \\ Pokfulam Road, Hong Kong \\ Email: \{sdma,nwong,tsng\}@eee.hku.hk
}

\begin{abstract}
In this paper, a time domain equalization algorithm is proposed for single-TX multiple-RX OFDM systems over frequency selective fading channels. The algorithm, which cancels most of the intersymbol interference (ISI), utilizes the orthogonality of the IFFT matrix and the second order statistics of the received signals. The signals are then detected, with the aid of only four pilots, from the equalizer output. The number of pilots required in the proposed algorithm is less than that in existing algorithms and channel length estimation is not needed. In addition, the proposed algorithm is applicable to both the case where the channel length is shorter than or equal to the length of cyclic prefix (CP), and the case where the channel length is longer than the length of cyclic prefix which results in inter-block interference (IBI). Simulation results confirm the effectiveness of the proposed algorithm in both cases and indicate that it is more practical as there is no restriction on the channel and $C P$ lengths.
\end{abstract}

\section{INTRODUCTION}

OFDM (Orthogonal Frequency Division Multiplexing) technique is considered as the one that has most potential for next generation communications and has been widely used in digital audio broadcasting (DAB), digital video broadcasting (DVB), and broadband wireless local area networks (IEEE 802.11a) $[1,2]$. It can effectively mitigate the effects of multipath propagation on the frequency selective fading channels as long as the channel length is shorter than or equal to the length of the cyclic prefix (CP). In this case, the signals can be easily detected by a set of parallel persubcarrier signal detectors on flat fading channels [3]. However, when the channel length is longer than the length of $\mathrm{CP}$, orthogonality of each subcarrier will be destroyed and inter-block interference (IBI) occurs, rendering conventional algorithms [1, 3, 4] inapplicable. Different approaches [5-7] have been proposed to detect the signals of OFDM systems wherein the channel length is longer than that of CP. In [5, $6]$, a time domain equalizer is inserted to reduce the channel to a filter model with an order shorter than or equal to the length of CP. The existing per-subcarrier detection algorithm [3] can thus be applied to detect the signals. This time domain equalization can also be transferred to a frequency domain operation as in [7]. However, accurate channel length estimation, which is difficult in practice, is required in these algorithms.

In this paper, a time domain equalization algorithm without channel length estimation is proposed for OFDM systems over frequency selective fading channels. Most of the intersymbol interference (ISI) is cancelled by the time domain equalizer, which makes use of the orthogonality of the IFFT matrix and the second order statistics of the received signals. The signals are then detected from the output of the equalizer with the aid of only four pilots. The number of pilots required in the proposed algorithm is less than that required in existing algorithms $[1,3]$, which only consider the case where the channel length is shorter than or equal to the length of $\mathrm{CP}$ and require at least $N$ pilots (where $N$ is the number of symbols in one OFDM block). In addition, the proposed algorithm is applicable to both the case where the channel length is shorter than or equal to the length of CP, and the case where the channel length is longer than the length of $\mathrm{CP}$. Simulation results verify that the proposed algorithm performs well in both cases.

The rest of the paper is organized as follows. In Section II, the OFDM system model is introduced. The time domain equalization algorithm is presented in Section III. In Section IV, the performance of the proposed algorithm is demonstrated by simulation. Finally, Section V draws the conclusion.

\section{SYSTEM MODEL}

Consider a single-input multiple-output (SIMO) system with $M$ receive antennas. At the transmitter, the white signals with zero mean and unit variance are partitioned into blocks of $N$ frequency domain symbols as $\overrightarrow{\mathbf{b}}_{i}=\left[\begin{array}{llll}\vec{b}_{i}[0] & \vec{b}_{i}[1] & \cdots & \vec{b}_{i}[N-1]\end{array}\right]^{T}$, where $(\bullet)^{T}$ represents transpose operation. Each block is transformed into a time domain block using $N$-point IFFT as $\mathbf{b}_{i}=\mathbf{F}_{N} \overrightarrow{\mathbf{b}}_{i}$, where $\mathbf{b}_{i}=\left[\begin{array}{llll}b_{i}[0] & b_{i}[1] & \cdots & b_{i}[N-1]\end{array}\right]^{T}$, and $\mathbf{F}_{N}$ is the $N \times N$ IFFT matrix with the $(n+1, k+1)$ th entry being $e^{j 2 \pi n k / N} / \sqrt{N}, n, k \in\{0,1, N-1\}$. It is obvious that the FFT matrix is $\mathbf{F}_{N}^{*}$ and $\mathbf{F}_{N} \mathbf{F}_{N}^{*}=\mathbf{I}_{N}$, where $(\bullet)^{*}$ denotes conjugate transpose and $\mathbf{I}_{N}$ is the $N \times N$ identity matrix. A CP of length $D$ is inserted into $\mathbf{b}_{i}$ to generate the $i$ th transmitted signal block as $\mathbf{s}_{i}=\left[\begin{array}{llll}s_{i}[0] & s_{i}[1] & \cdots & s_{i}\left[N^{\prime}-1\right.\end{array}\right]^{T}$, in which $N^{\prime}=N+D$ and 


$$
s_{i}[n]=\left\{\begin{array}{c}
b_{i}[n-D+N], 0 \leq n \leq D-1 \\
b_{i}[n-D], \mathrm{D} \leq n \leq N^{\prime}-1
\end{array} .\right.
$$

The time domain blocks $\mathbf{s}_{i}$ are then serially transmitted over frequency selective fading channels. Denote the frequency selective channel between the transmit antenna and the $m$ th receive antenna as $h_{m}(l)$, which is modeled as an $L_{m}$ th-order FIR filter. Let $L=\max _{1 \leq m \leq M}\left(L_{m}\right)$. The received $i$ th block at the $m$ th receive antenna is therefore

$$
\begin{gathered}
y_{i, m}[n]=\sum_{l=0}^{L} h_{m}(l) s_{i}[n-l]+w_{i, m}[n] \\
n=0,1, \cdots, N^{\prime}-1, \quad m=1,2, \cdots, M,
\end{gathered}
$$

where $h_{m}(l)$ is zero-padded for $L_{m}<l \leq L$ $s_{i}[n]=s_{(i-1)}\left[N^{\prime}+n\right]$ for $n<0, \quad s_{i}[n]=s_{(i+1)}\left[n-N^{\prime}\right]$ for $n \geq N^{\prime}$, and $w_{i, m}[n]$ is the independently identically distributed (i.i.d.) noise, which is uncorrelated with the transmitted signals. Note that there is no restriction on the channel length and the length of CP. By defining

$$
\begin{gathered}
\mathbf{y}_{i}[n]=\left[\begin{array}{llll}
y_{i, 1}[n] & y_{i, 2}[n] & \cdots & y_{i, M}[n]
\end{array}\right]^{T}, \\
\mathbf{h}(l)=\left[\begin{array}{llll}
h_{1}(l) & h_{2}(l) & \cdots & h_{M}(l)
\end{array}\right]^{T}, \\
\mathbf{w}_{i}[n]=\left[\begin{array}{llll}
w_{i, 1}[n] & w_{i, 2}[n] & \cdots & w_{i, M}[n]
\end{array}\right]^{T},
\end{gathered}
$$

we can express (2) in vector form as

$$
\mathbf{y}_{i}[n]=\sum_{l=0}^{L} \mathbf{h}(l) s_{i}[n-l]+\mathbf{w}_{i}[n], \quad n=0,1, \cdots, N^{\prime}-1,
$$

Collecting the $N^{\prime}$-sampled received signal as

$$
\begin{gathered}
\mathbf{y}_{i}^{(J)}=\left[\begin{array}{lll}
\mathbf{y}_{i}[-J]^{T} & \mathbf{y}_{i}[-J+1]^{T} \quad \cdots \quad \mathbf{y}_{i}\left[N^{\prime}-1-J\right]^{T}
\end{array}\right]^{T}, \\
J=0, \pm 1, \pm 2, \cdots,
\end{gathered}
$$

where $\quad \mathbf{y}_{i}[n]=\mathbf{y}_{i-1}\left[N^{\prime}+n\right] \quad$ for $n<0, \quad$ and $\mathbf{y}_{i}[n]=\mathbf{y}_{i+1}\left[n-N^{\prime}\right]$ for $n \geq N^{\prime}$, we obtain

$$
\mathbf{y}_{i}^{(J)}=\mathbf{H x}_{i}^{(J)}+\mathbf{w}_{i}^{(J)}, \quad J=0, \pm 1, \pm 2, \cdots
$$

Here

$$
\begin{gathered}
\mathbf{x}_{i}^{(J)}=\left[\begin{array}{llllll}
s_{i}[-L-J] & \cdots & s_{i}[0] & \cdots & s_{i}\left[N^{\prime}-1-J\right]
\end{array}\right]^{T}, \\
\mathbf{w}_{i}^{(J)}=\left[\begin{array}{llllll}
\mathbf{w}_{i}[-J]^{T} & \mathbf{w}_{i}[-J+1]^{T} & \cdots & \mathbf{w}_{i}\left[N^{\prime}-1-J\right]^{T}
\end{array}\right]^{T}, \\
\mathbf{H}=\left[\begin{array}{ccccccc}
\mathbf{h}(L) & \mathbf{h}(L-1) & \cdots & \mathbf{h}(0) & \mathbf{0} & \cdots & \mathbf{0} \\
\mathbf{0} & \mathbf{h}(L) & \mathbf{h}(L-1) & \cdots & \mathbf{h}(0) & \ddots & \mathbf{0} \\
\vdots & \ddots & \ddots & \ddots & \ddots & \ddots & \vdots \\
\mathbf{0} & \cdots & \mathbf{0} & \mathbf{h}(L) & \mathbf{h}(L-1) & \cdots & \mathbf{h}(0)
\end{array}\right] .
\end{gathered}
$$

A general assumption that the channel convolution matrix $\mathbf{H}$ is of full column rank after removing all-zero columns is made here. In practice, when the number of receive antennas $M$ satisfies that $M>\left(L+N^{\prime}\right) / N^{\prime}$, the matrix $\mathbf{H}$ has more rows than columns. Hence, it is most likely of full column rank. Note that the received signal vector $\mathbf{y}_{i}^{(J)}$ is the $i$ th received block signal vector after shifting $J$ samples. It contains both the $(i-1)$ th and the $i$ th block signals.

When $0 \leq J \leq N-L$, the signal vector $\mathbf{x}_{i}^{(J)}$ in (9) can be represented with respect to the frequency domain signal as $\mathbf{x}_{i}^{(J)}=\mathbf{F}_{2 N}^{(J)} \overrightarrow{\mathbf{c}}_{i}$, where $\overrightarrow{\mathbf{c}}_{i}=\left[\begin{array}{ll}\overrightarrow{\mathbf{b}}_{(i-1)}^{T} & \overrightarrow{\mathbf{b}}_{i}^{T}\end{array}\right]^{T}$, and

$$
\mathbf{F}_{2 N}^{(J)}=\left[\begin{array}{cc}
\mathbf{F}_{N}(N-L-J+1: N) & \mathbf{0} \\
\mathbf{0} & \mathbf{F}_{N}(N-D+1: N) \\
\mathbf{0} & \mathbf{F}_{N}(1: N-J)
\end{array}\right] .
$$

In (12), $\mathbf{F}_{N}(a: b)$ denotes a submatrix of $\mathbf{F}_{N}$, composed by the rows between the $a$ th row and the $b$ th row of $\mathbf{F}_{N}$. It follows that the received signal vector $\mathbf{y}_{i}^{(J)}$ can also be rewritten as

$$
\begin{gathered}
\mathbf{y}_{i}^{(J)}=\mathbf{H} \mathbf{x}_{i}^{(J)}+\mathbf{w}_{i}^{(J)}=\mathbf{H F}_{2 N}^{(J)} \overrightarrow{\mathbf{c}}_{i}+\mathbf{w}_{i}^{(J)}, \\
0 \leq J \leq N-L .
\end{gathered}
$$

\section{TIME DEMAIN EQUALIZATION}

In this section, a time domain equalization algorithm without channel length estimation is proposed for OFDM systems on frequency selective fading channels. The algorithm not only works in the case where the channel length is shorter than or equal to the $\mathrm{CP}$ length, it is also applicable to the case where the channel length is longer than the $\mathrm{CP}$ length. To simplify the derivation of the algorithm, zero noise is first assumed. The effect of noise on the developed algorithm is then examined. In the absence of noise, $\mathbf{y}_{i}^{(J)}$ can be expressed as

$$
\begin{gathered}
\mathbf{y}_{i}^{(J)}=\mathbf{H} \mathbf{x}_{i}^{(J)}, J=0, \pm 1, \pm 2, \cdots \\
\mathbf{y}_{i}^{(J)}=\mathbf{H x}_{i}^{(J)}=\mathbf{H F}_{2 N}^{(J)} \overrightarrow{\mathbf{c}}_{i}, 0 \leq J \leq N-L .
\end{gathered}
$$

Consider the auto-correlation of the received signal vector $\mathbf{y}_{i}^{(J)}$ as

$$
\begin{aligned}
\mathbf{R}_{y}^{(J)} & =E\left\{\mathbf{y}_{i}^{(J)} \mathbf{y}_{i}^{(J)^{*}}\right\}=\mathbf{H} \mathbf{F}_{2 N}^{(J)} E\left\{\overrightarrow{\mathbf{c}}_{i} \overrightarrow{\mathbf{c}}_{i}^{*}\right\} \mathbf{F}_{2 N}^{(J) *} \mathbf{H}^{*}, 0 \leq J \leq N-L . \\
& =\mathbf{H F}_{2 N}^{(J)} \mathbf{F}_{2 N}^{(J) *} \mathbf{H}^{*}
\end{aligned}
$$

Based on the orthogonality of the IFFT matrix $\left(\mathbf{F}_{N} \mathbf{F}_{N}^{*}=\mathbf{I}_{N}\right)$, the matrix $\mathbf{F}_{2 N}^{(J)}$ (12) satisfies

$$
\mathbf{F}_{2 N}^{(D)} \mathbf{F}_{2 N}^{(D)^{*}}=\left[\begin{array}{ccc}
\mathbf{I}_{L+D} & \mathbf{0} & \mathbf{0} \\
\mathbf{0} & \mathbf{I}_{D} & {\left[\begin{array}{cc}
\mathbf{0} & \mathbf{I}_{D-D} \\
\mathbf{0} & \mathbf{0}
\end{array}\right]} \\
\mathbf{0}\left[\begin{array}{rr}
\mathbf{0} & \mathbf{0} \\
\mathbf{I}_{D-D} & \mathbf{0}
\end{array}\right] & \mathbf{I}_{N-D}
\end{array}\right]=\mathbf{I}_{N^{\prime}+L},
$$

where $\mathbf{U}$ is an $\left(N^{\prime}+L\right) \times\left(N^{\prime}+L\right)$ matrix with only 2 entries having values of one at the positions $(L+1, N+L+1)$ and 
( $N+L+1, L+1)$, while all remaining entries are zeros. Applying (17) and (18) into (16), we obtain

$$
\begin{gathered}
\mathbf{R}_{y}^{(0)}-\mathbf{R}_{y}^{(1)}=\mathbf{H}\left(\mathbf{F}_{2 N}^{(0)} \mathbf{F}_{2 N}^{(0)^{* *}}-\mathbf{F}_{2 N}^{(1)} \mathbf{F}_{2 N}^{(1)^{* *}}\right) \mathbf{H}^{* *}=\mathbf{H} \mathbf{U H}^{* \prime}, \\
\mathbf{R}_{y}^{(D)}=\mathbf{H} \mathbf{H}^{\prime \prime} .
\end{gathered}
$$

Now, an equalizer can be designed using the secondorder statistics of the received signal vector as

$$
\mathbf{G}=\left(\mathbf{R}_{y}^{(0)}-\mathbf{R}_{y}^{(1)}\right) \mathbf{R}_{y}^{(D) \#}=\mathbf{H U H}^{*}\left(\mathbf{H H}^{*}\right)^{\#} .
$$

in which $(\bullet)^{\#}$ stands for pseudo-inverse. Applying the equalizer to the received signal vector $\mathbf{y}_{i}^{(J)}(14)$, it yields

$$
\begin{gathered}
\mathbf{o}_{i}^{(J)}=\mathbf{G} \mathbf{y}_{i}^{(J)}=\mathbf{H U H}^{*}\left(\mathbf{H} \mathbf{H}^{*}\right)^{\#} \mathbf{H} \mathbf{x}_{i}^{(J)}, \\
J=0, \pm 1, \pm 2, \cdots .
\end{gathered}
$$

Under the assumption that the channel convolution matrix $\mathbf{H}$ is of full column rank after removing all-zero columns, it satisfies [8] that $\mathbf{H}^{*}\left(\mathbf{H H}^{*}\right)^{\#} \mathbf{H}=\mathbf{I}_{\left(N^{\prime}+L\right) P}^{H}$ in which $\mathbf{I}_{N^{\prime}+L}^{H}$ is an $\left(N^{\prime}+L\right) \times\left(N^{\prime}+L\right)$ identity matrix with all-zero rows corresponding to the all-zero columns of $\mathbf{H}$. It follows

$$
\overrightarrow{\mathbf{o}}_{i}^{(J)}=\mathbf{H U} \overrightarrow{\mathbf{x}}_{i}^{(J)}=\mathbf{H}_{p a r t} \overrightarrow{\mathbf{x}}_{i, p a r t}^{(J)},
$$

where

$$
\begin{aligned}
& \mathbf{H}_{\text {part }}=\left[\begin{array}{ll}
\mathbf{H}(N+L+1) & \mathbf{H}(L+1)
\end{array}\right], \\
& \mathbf{x}_{i, p a r t}^{(J)}=\left[s_{i}[-J] \quad s_{i}[N-J]\right]^{T} .
\end{aligned}
$$

In (24), $\mathbf{H}(a)$ denotes the $a$ th column of $\mathbf{H}$. It is apparent that most ISI is cancelled by the equalizer $\mathbf{G}$, while retaining the 2 preselected columns of the channel convolution matrix. Namely, the signal vector $\mathbf{o}_{i}^{(J)}$ contains only 2 path signals.

In order to recover the transmitted signals from $\mathbf{o}_{i}^{(J)}$, knowledge of the matrix $\mathbf{H}_{\text {part }}$ is required. For better performance, it is preferred to use some pilots to estimate $\mathbf{H}_{\text {part }}$. As the matrix $\mathbf{H}_{\text {part }}$ has only 2 columns, 4 pilot symbols are enough, namely,

$$
\hat{\mathbf{H}}_{\text {part }}=\mathbf{O}_{i, \text { pilot }} \mathbf{X}_{i, \text { pilot }}^{*}\left(\mathbf{X}_{i, \text { pilot }} \mathbf{X}_{i, \text { pilot }}^{*}\right)^{\#},
$$

where $\mathbf{O}_{i, \text { pilot }}=\left[\begin{array}{ll}\mathbf{o}_{i}^{(0)} & \mathbf{o}_{i}^{(1)}\end{array}\right]$, and $\mathbf{X}_{i, \text { pilot }}$ consists of the 4 pilot symbols as $\mathbf{X}_{i, \text { pilot }}=\left[\begin{array}{ll}\mathbf{x}_{i, \text { part }}^{(0)} & \mathbf{x}_{i, \text { part }}^{(1)}\end{array}\right]$. The number of pilots required in the proposed algorithm is less than that required in existing algorithms [1,3], which only consider the case where the channel length is shorter than or equal to the length of $\mathrm{CP}$ and require at least $N$ pilots.

With the estimated matrix $\hat{\mathbf{H}}_{\text {part }}$, the partial transmitted signal vector $\mathbf{x}_{i, \text { part }}^{(J)}$ can be detected based on least-squares criteria as in [8]

$$
\hat{\mathbf{x}}_{i, p a r t}^{(J)}=\left(\mathbf{H}_{\text {part }}^{*} \mathbf{H}_{\text {part }}\right)^{\#} \mathbf{H}_{\text {part }}^{* *} \mathbf{o}_{i}^{(J)} .
$$

Since the signal vector $\hat{\mathbf{x}}_{i, \text { part }}^{(J)}$ contains 2 path signals $s_{i}[-J]$ and $s_{i}[N-J]$, any path signal with the parameter $J$ having different values can be utilized to estimate the frequency domain signal $\vec{b}_{i}[n]$ by FFT operation. Note that knowledge of the channel length is not required in the proposed algorithm. It turns out that channel length estimation is not needed in the proposed algorithm, while it is needed in the existing algorithms [5-7].

So far the derivation is based on zero-noise assumption. When additive white channel noise with variance $\sigma^{2}$ is present, the auto-correlation matrix of $\mathbf{y}_{i}^{(J)}$ is

$$
\begin{gathered}
\mathbf{R}_{y}^{(J)}=E\left\{\mathbf{y}_{i}^{(J)} \mathbf{y}_{i}^{(J)^{*}}\right\}=\mathbf{H F}_{2 N}^{(J)} \mathbf{F}_{2 N}^{(J)^{*}} \mathbf{H}^{*}+\sigma^{2} \mathbf{I}_{M N^{\prime}}, \\
0 \leq J \leq N-L .
\end{gathered}
$$

The noise contribution can be subtracted when the noise variance $\sigma^{2}$ is known. When $\sigma^{2}$ is unknown, it can be estimated from the singular value decomposition of $\mathbf{R}_{y}^{(D)}$ where $\mathbf{R}_{y}^{(D)}=\mathbf{H H}^{*}+\sigma^{2} \mathbf{I}_{M N^{\prime}}$. Since some error generally exists in the estimation of $\sigma^{2}$ and this error will degrade the performance, it is preferred not to subtract the noise contribution from $\mathbf{R}_{y}^{(J)}$ in our implementation. Instead, the equalizer $\mathbf{G}$ is constructed based on $\mathbf{R}_{y}^{(J)}$ as if it were noiseless. As the partial transmitted signal detection is performed based on some pilot symbols, the noise contribution has little impact on the overall performance.

\section{Simulation Results}

Computer simulations are conducted to investigate the performance of the proposed algorithm. In the following examples, an OFDM system with $M=2$ receive antennas is considered. The OFDM parameters are: $N=16$ and $D=4$. The transmitted signals are modulated by QPSK scheme. The frequency selective fading channel responses are randomly generated. The performance measure, BER (bit error rate), is computed by averaging the Monte Carlo test results on $3 \times 10^{5}$ OFDM blocks.

First, the case $L=3$ is considered. As the channel length ( $L=3$ ) is shorter than the length of $\mathrm{CP}(D=4)$, the conventional per-subcarrier detection algorithm [3] can be applied. Both the conventional algorithm [3] using LeastSquares criteria and the MMSE signal detection algorithm with the exact channel state information (MMSE-ideal, with $\left.\mathbf{G}_{\text {MMSE-ideal }}=\mathbf{H}^{*}\left(\mathbf{H H}^{*}+\mathbf{I} / S N R\right)^{-1}\right)$ are implemented for comparison. Fig. 1 shows the BER performance of various algorithms for the case $L=3$. It is obvious that the proposed algorithm is superior to the conventional algorithm over the range of SNR considered. Note that the performance of the MMSE-ideal algorithm can be considered as a lower bound. Next, the case $L=6$ is considered. Only the MMSE-ideal algorithm and the proposed algorithm are implemented as the conventional algorithm is inapplicable when the channel 
length is longer than the length of $\mathrm{CP}$, which destroys the orthogonality of subcarriers resulting in IBI. Results are shown in Fig. 2. It indicates the proposed algorithm also performs well when the channel length is longer than the length of CP. To illustrate the impact of the channel length and the $\mathrm{CP}$ length on the proposed algorithm, the performance for $L=3,4,5,6$ cases is shown in Fig. 3 . It is apparent that the performance only degrades slightly when the channel length increases from $3(L<D)$ to $6(L>D)$. It demonstrates that the proposed algorithm is robust against the channel length and is more practical as there is no restriction on the channel length and the $\mathrm{CP}$ length.

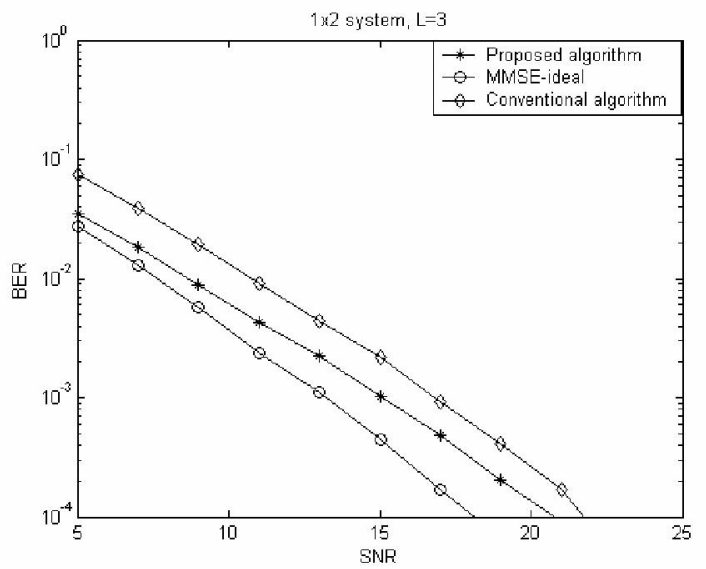

Fig. 1 BER versus SNR for the $L=3$ case

\section{CONCLUSION}

This paper has proposed a time domain equalization algorithm for SIMO OFDM systems without channel length estimation. The proposed equalizer cancels most of the ISI using the orthogonality of the IFFT matrix and second order statistics. Only 4 pilot symbols are required to detect the transmitted signals from the equalizer output. The number of pilots required is less than that required in the existing algorithm and channel length estimation is not needed. Simulation results have verified that the proposed algorithm performs well in both the cases where the channel length is longer than, or shorter than/equal to the length of $\mathrm{CP}$. Consequently, the proposed algorithm constitutes a more practical solution.

\section{ACKNOWLEDGMENT}

This work was supported by the Hong Kong Research Grants Council (Grant No. 7168/03E).

[1] B. Muquet, Z. Wang, G. B. Giannakis, M. de Courville, and P. Duhamel, "Cyclic prefixing or zero padding for wireless multicarrier transmissions?", IEEE Trans. on Commun., vol. 50, pp. 2136-2148, Dec. 2002

[2] J. Lei and T.S. Ng, "A consistent OFDM carrier frequency offset estimator based on distinctively spaced pilot tones", IEEE Trans. on Wireless Commun., vol. 3, pp. 588-599, Mar. 2004
[3] M. Speth, S.A. Fechtel, G. Fock, and H. Meyr, "Optimum receiver design for wireless broad-band systems using OFDM-Part I", IEEE Trans. on Commun., vol. 47, pp. 1668-1677, N̉ov. 1999

[4] M. Luise, R. Reggiannini, and G.M. Vitetta, "Blind equalization/detection for OFDM signals over frequency-selective channels", IEEE J. Select. Areas in Commun., vol. 16, pp. 1568-1578, Oct. 1998

[5] P.J.W. Melsa, R.C. Younce, and C.E. Rohrs, "Impulse response shortening for discrete multitone transceivers", IEEE Trans. on Commun., vol. 44, pp. 1662-1672, Dec. 1996

[6] N. Al-Dhahir, and J. Cioffi, "Optimum finite-length equalization for multicarrier transceivers", IEEE Trans. on Commun., vol. 44, pp. 56-64, Jan. 1996

[7] K. Van Acker, G. Leus, M. Moonen, O. van de Wiel, and T. Pollet, "Per-tone equalization for DMT-based systems", IEEE Trans. on Commun., vol. 49, pp. 109119 , Jan. 2001

[8] S. M. Kay, Fundamentals of Statistical Signal Processing: Estimation Theory, New Jersey: PrenticeHall, 1993

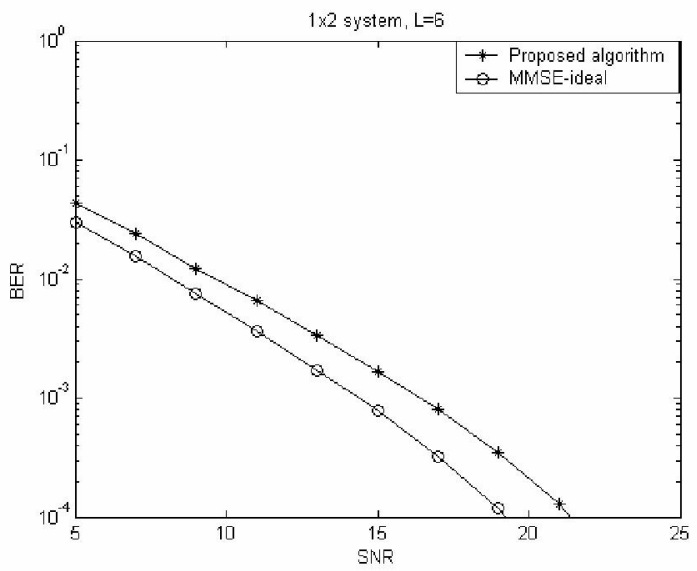

Fig. 2 BER versus SNR for the $L=6$ case

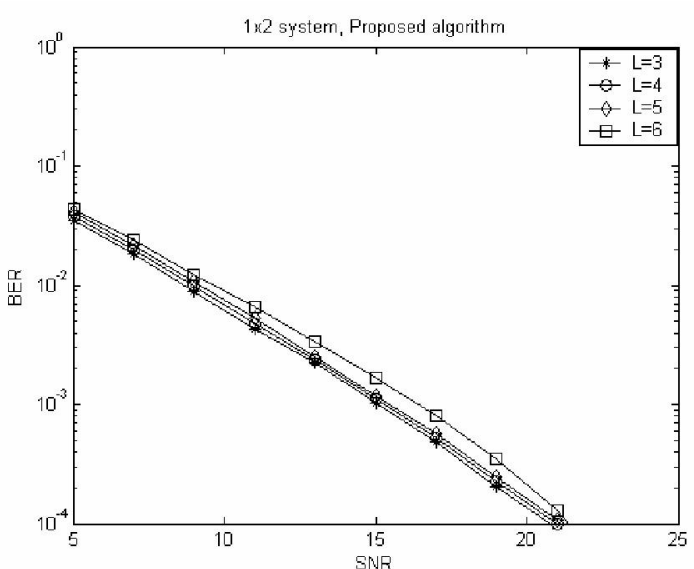

Fig. 3 BER versus SNR for the cases $L=3,4,5,6$

$$
(D=4)
$$

